\title{
Vom Sternerestaurant in die Kantine? Möglichkeiten und Grenzen für ein berufliches Altern im Gastgewerbe
}

K. Gublemann und C. Best

\section{Abstract}

Gesundes Altern in Arbeit, im demografischen Wandel wichtiger denn je, ist keineswegs in allen Berufen gleich gut möglich. In jugendzentrierten Branchen wie dem hier untersuchten Gastgewerbe fehlt es noch immer an Karrierewegen, bei denen die sich wandelnden Bedürfnisse der Beschäftigten mit den Arbeitsbedingungen in Einklang gebracht werden können. Der Beitrag zeigt in einer biografiezentrierten Sichtweise die Passungsprobleme auf, die ab den mittleren Lebensphasen offensichtlich werden und häufig zu Branchenausstiegen oder beruflichen Diskontinuitäten führen und beschreibt Ansätze zu deren Lösung. Die Befunde verdeutlichen die Relevanz einer biografischen Perspektive für eine lebensphasengerechte Arbeitsgestaltung, insbesondere in Berufen mit begrenzter Tätigkeitsdauer.

\section{Einleitung - der demografische Wandel - kein Thema mehr?}

Die Arbeitswelt ist aktuell sowohl gekennzeichnet von tiefgreifenden Veränderungen im Rahmen von Digitalisierung, Globalisierung und Flexibilisierung, die Betriebe vor große Herausforderungen hinsichtlich der Arbeitsgestaltung stellen, als auch vom branchenspezifisch differierenden Fehlen von Fachkräften (Dettmann et al. 2019). In dieser Gemengelage stellt der demografische Wandel (siehe u.a. die Beiträge von Bellmann und Langhoff in diesem Band) weitere Anforderungen an Arbeitsgestaltung (Frerichs 2019, S. 894), die nicht in allen Branchen gleich gut gelöst werden (können). Zwar ist Altern in Arbeit nicht nur durch die gesetzlich angelegte Verlängerung der Lebensarbeitszeit und Tendenzen steigender Altersarmut eine Notwendigkeit für die Beschäftigten, sondern wie die Ergebnisse des Mikrozensus zur Erwerbsbeteiligung der über 55-Jährigen dokumentieren, zunehmend Realität ${ }^{1}$. Jedoch zeigen die Zahlen des Mikrozensus neben wesentlichen Geschlechterunterschieden sehr deutlich, dass einige Branchen nach wie vor nur geringe Anteile älterer Beschäftigter auf- 
weisen (Statistisches Bundesamt 2019, S. 370). Eine dieser Branchen ist das jugendzentrierte Gastgewerbe - im Vergleich aller Branchen verzeichnen nur Arbeitnehmerüberlassungen einen noch geringeren Wert an Beschäftigten über 55 (Statistik der Bundesagentur für Arbeit 2019). Durch wachsenden Nachwuchsmangel stellt sich aber auch für das Gastgewerbe die drängende Frage nach Möglichkeiten und Grenzen der Bewahrung von Gesundheit und Leistungsfähigkeit in der Arbeit. Konkret soll daher nachfolgend untersucht werden, welche Faktoren ein Älterwerden in einem gastgewerblichen Beruf behindern und welche Möglichkeiten es zu deren Überwindung gibt. Die bereits 2000 auf dem Kongress „Arbeit und Altern" konstatierte Diversifizierung der Lebensphasen in ihrer Implikation für die arbeitsbezogenen Bedürfnisse der Beschäftigten einerseits und die daraus resultierenden Anforderungen an alternsgerechte Arbeitsgestaltung andererseits (Naegele 2000) sind Anlass der im vorliegenden Beitrag zum Tragen kommenden biografischen Sichtweise. Nach einem kurzen Überblick über wissenschaftliche Erkenntnisse zum Themenspektrum Altern in Arbeit kontrastiert der Artikel die Situation im Gastgewerbe mit den Anforderungen vonseiten der Beschäftigten. In einem Abgleich beider Seiten werden Probleme für ein Älterwerden im Beruf und Ansätze für einen längeren Berufsverbleib herausgearbeitet.

\section{Altern in Arbeit und lebensphasengerechte Arbeitsgestaltung}

Mit dem Anstieg der allgemeinen Lebenserwartung stellt sich auch die Frage nach einer (Neu)-definition des Alter(n)s. Die daraus resultierende pluralistische Betrachtung des Alters als (autonome) Lebensphase berücksichtigt in einer Abwendung der Verknüpfung mit dem Renteneintritt in stärkerem Maße die Individualität und Prozesshaftigkeit des Alters, da neben Erwerbsbiografien auch Rentenübergänge brüchiger und ausgedehnter werden. Arbeit im Alter gewinnt somit an Bedeutung, da sie entweder aus ökonomischen Gesichtspunkten nötig wird oder der Gestaltung der Lebensphase im Alter dient (Schneider \& Stadelbacher 2019, S. 3ff.).

Die Veränderungen in der Wahrnehmung und Bedeutung des Alter(n)s spiegeln sich notwendigerweise auch in der Arbeitsmarkt- und Rentenpolitik wider. Waren die 1990er-Jahre noch durch die Entlastung des Arbeitsmarktes mittels vorzeitiger Verrentung älterer Beschäftigter geprägt, zielen Arbeitsmarkt- und arbeitspolitische Reformen nunmehr auf die längere Beschäftigung Älterer (Kistler et al. 2019, S. 90). Dies zeigt sich neben der o.g. stärkeren Erwerbsbeteiligung der über 55-Jährigen auch in einem deutlichen Anstieg des Rentenzugangsalters bei Altersrenten von 62 Jah- 
ren im Jahr 2000 auf 64 Jahre 2018 (Deutsche Rentenversicherung 2019, S. 19).

Varekamp et al. (2013, S. 409) belegten, basierend auf der GEDA-Studie 2009/2010 jedoch, dass der längere Verbleib im Beruf bei Beschäftigten im Alter von 55-64 Jahren nicht zwangsläufig unter guter Gesundheit geschieht: Rund $50 \%$ aller erwerbstätigen Männer und $38 \%$ aller erwerbstätigen Frauen jener Altersgruppe wiesen zwei oder mehr Erkrankungen auf, rund $50 \%$ Frauen und $63 \%$ Männer je eine Erkrankung (Hasselhorn \& Ebener 2018, S. 216). Gleichzeitig lag die Beschäftigtenquote von über 55jährigen Personen ohne Erkrankungen bei Männern um $22 \%$ und bei Frauen um $29 \%$ unter der der jüngeren Kohorte (Varekamp et al. 2013). Eindeutige Aussagen über die Erwerbsteilhabe älterer Menschen im Zusammenhang mit ihrer Gesundheit lassen sich also nur schwer treffen.

Angesichts der hinreichend beschriebenen Hintergründe, die eine längere Beschäftigungsfähigkeit erfordern (siehe u.a. die Beiträge von Naegele und Hess sowie von Anlauft und Feldes in diesem Band), wurde frühzeitig der Bedarf von alternsgerechter Arbeitsgestaltung erkannt (z.B. Frerichs 2019). Arbeitsfähigkeit ergibt sich aus dem Zusammenhang aus individuellen Ressourcen und den Anforderungen der Arbeitstätigkeit und wird bei älteren Arbeitnehmer*innen durch ein ungünstiges Zusammenspiel beider Seiten auf den Ebenen Gesundheit, Qualifikation und Motivation erschwert (Naegele 2005). Diese Passungsprobleme entstehen nicht plötzlich, sondern sind das Ergebnis kumulierter biografischer Ereignisse, Verhaltensweisen und Anforderungen. Um einen möglichst langen und gesunden Verbleib im Berufsleben zu ermöglichen, bedarf es daher einer alternsgerechten Arbeitsgestaltung, die nicht erst im „Alter“2 oder bei auftretenden gesundheitlichen Problemen beginnt, sondern über die gesamte Berufsbiografie hinweg den sich wandelnden Bedürfnissen der Beschäftigten angepasst ist (Kistler 2008, S. 40). Es gilt Personen mit guter Gesundheit länger im Beruf zu halten, Personen in Berufen mit höheren Belastungen und Gefährdungen präventiv zu unterstützen: bspw. Laufbahnperspektiven aufzuzeigen und Personen mit schlechterer Gesundheit zu entlasten. Hierfür ist auf betrieblicher Ebene die Anerkennung gefordert, dass sich die Bedürfnisse der Beschäftigten und ihre Belastbarkeit sowohl in Bezug auf die Arbeit als auch im Bereich der Lebenswelt von Lebensphase zu Lebensphase ändern, was deren Vereinbarkeit sowohl positiv als auch negativ beeinflussen kann ${ }^{3}$. Ein betriebliches systemisches Alter(n)smanagement, welchem ein integratives Verständnis innewohnt, das Handlungsfelder wie "Gesundheitsförderung, Weiterbildung, Arbeitsgestaltung, Wissensmanagement, Personalentwicklung und -rekrutierung unter Beachtung von Gesichtspunkten der Vereinbarkeit mit lebensweltlichen Pflich- 
ten und -aufgaben [sic]" (Brandl et al. 2020, S. 119) berücksichtigt und sich lebensphasengerecht darstellt, könnte eine vielversprechende Lösung für die bekannten Problemlagen sein. An dieser Stelle müssen jedoch auch gänzlich disgruente Bedarfslagen von Privatem und Beruflichem anerkannt werden, die einen Berufswechsel bedingen. Fraglich ist an dieser Stelle, inwieweit die bereits vorhandenen Ansätze für einen längeren Berufsverbleib auf die Veränderung von Bedürfnissen Beschäftigter im Lebensverlauf reagieren.

\section{Altern im Gastgewerbe - ein unmögliches Unterfangen?}

Basierend auf den genannten Erkenntnissen zum Altern in Arbeit wird im Folgenden das Altern im Gastgewerbe untersucht, einer Branche, die traditionell jugendzentriert ist und bislang wenig Optionen für bzw. Bemühungen um eine alternsgerechte Arbeitsgestaltung bietet. In diesem Kapitel stehen die Aspekte Altern in Gesundheit am Arbeitsplatz sowie der Prozess sich im Altersgang wandelnder Bedürfnisse der Beschäftigten im Fokus.

\subsection{Altern im Gastgewerbe - das Vorbildpotenzial von Kantinen und Campingplätzen}

Das Gastgewerbe gehört mit einem Jahresumsatz von rund $94 \mathrm{Mrd}$. $€$ und 2,4 Mill. Beschäftigten (DEHOGA 2020) zu den wichtigsten Wirtschaftszweigen der Bundesrepublik. Die Arbeit im Gastgewerbe ist so facettenreich wie die in der Branche versammelten Betriebstypen, unter denen neben einigen umsatzstarken Ketten die (häufig inhabergeführten) Klein- und Kleinstbetriebe dominieren. Unterschieden wird grundsätzlich in Beherbergungsbetriebe mit oder ohne Speisen- und Getränkeangebot und die reine speisen- und getränkeorientierte Gastronomie. Die Heterogenität der Branche legt einen genaueren Blick auf die Branchenspezifika und die Altersverteilung in den verschiedenen Betriebstypen der Branche nahe.

Bereits in der Beschäftigtenstruktur des gesamten Gastgewerbes zeigen sich starke Unterschiede zur Gesamtwirtschaft: Im Gastgewerbe sind neben jungen Beschäftigten mitarbeitende Inhaber*innen, Frauen, Beschäftigte mit Zuwanderungsgeschichte, Beschäftigte ohne formale Berufsqualifikation und geringfügig Beschäftigte überrepräsentiert (BA 2019, S. 12; Statistisches Bundesamt 2019). Der Nachwuchs- und Fachkräftemangel hat 
in den letzten Jahren stetig zugenommen: Die Ausbildungszahlen sind seit ihrem Höchststand im Jahr 2007 um 52\% gesunken, und 56\% der ausbildenden Betriebe konnten nicht alle Ausbildungsplätze besetzen (DEHOGA 2020; DIHK 2019, S.7). Hinzu kommt eine starke Fluktuation, die sich in einem Fluktuationskoeffizienten von 0,696 manifestiert ${ }^{4}$ (BA 2018, S. 140) und bereits in der Ausbildung beginnt, wovon Lösungsquoten zeugen, die mit 40-50 \% unter den höchsten aller Branchen sind (BIBB 2020). Auch wenn vonseiten des Verbandes betont wird, dass darunter auch Ausbildungsstellenwechsel und nicht bestandene Prüfungen fallen und dies keinesfalls ein reiner Beleg für Schwächen in der Ausbildungsqualität sei (DEHOGA 2018), landen die Ausbildungsbetriebe des Gastgewerbes seit vielen Jahren im DGB Ausbildungsreport auf den letzten Plätzen, wobei die Ausbildungsqualität in kleineren Betrieben generell schlechter eingeschätzt wird als in größeren (DGB Bundesvorstand 2019, Guhlemann et al. 2016). Abgesehen von der unzureichenden Vermittlung der Ausbildungsinhalte zugunsten der Nutzung von Auszubildenden als kostengünstige Arbeitskräfte, stehen in diesem Zusammenhang häufig die Arbeitsbedingungen in der Kritik: hier besonders die ungünstige Lage und schlechte Planbarkeit der Arbeitszeiten, viele Überstunden, körperliche Belastungen und Stress, Führungsmängel und die niedrige Entlohnung (ebd.).

Die problematischen Arbeitsbedingungen können in einen direkten Zusammenhang zu den genannten Merkmalen der Branche gestellt werden: Das stark nachfragegebundene Arbeitsaufkommen bestimmt die Arbeits- und Einsatzzeiten des Personals; die Planbarkeit der Arbeitszeiten ist damit eng an die Vorhersehbarkeit der Kundenströme gekoppelt. Obwohl auch etablierte, größere Betriebe, ruhigere' Tage haben, stellt dieser Umstand kleinere inhabergeführte Betriebe, unter denen Konkurrenz und Fluktuation hoch sind, vor besondere Herausforderungen. Verbunden mit der durch Konkurrenz und Fachkräftemangel dünnen Personaldecke führt die Orientierung an den Gästewünschen hier häufig zur Übertragung des Selbstständigenhabitus auf das Personal, von denen eine Anpassung der Arbeitsbereitschaft an die Nachfrage ebenso erwartet wird wie (meist unvergütete) Mehrarbeit. Unplanbare Nachfragespitzen und eine dünne Personaldecke führen zum Stresserleben in der Arbeit, das dazu beiträgt, körperliche Belastungen schlechter kompensieren zu können. Führungsmängel hingegen ergeben sich zum einen aus der starken Belastung der Führungskräfte, der hierarchisch geprägten Branchenkultur und der Besetzung von Führungspositionen nach Fach- anstelle von Führungskompetenz (Krüger \& Guhlemann 2018).

Dass Arbeit in späteren Lebensphasen im Gastgewerbe vor diesem Hintergrund schlechter möglich ist als in anderen Branchen, zeigen die Zah- 
len des Statistischen Bundesamtes: Unter den Beschäftigten sind lediglich $14 \%$ über 55 Jahre alt, während die unter 25-Jährigen überrepräsentiert sind. Bereits für den Zeitraum 2000 bis 2014 zeigten Schlote-Sautter et al. (2018), dass der durch den demografischen Wandel bedingte Altersanstieg der Gesamtbeschäftigten im Gastgewerbe wesentlich geringer ausfiel. Dieser Trend setzt sich bis heute fort. Während in der Gesamtwirtschaft der Anteil der über 55-Jährigen von 16,5\% auf $21 \%$ gestiegen ist, verzeichnet das Gastgewerbe lediglich einen Anstieg von 13,2\% auf 15,8\%. Ein genauerer Blick auf die Zahlen (Statistisches Bundesamt 2019) verdeutlicht jedoch, dass in einigen Betriebstypen Arbeit im Alter von über 55 Jahren keine Seltenheit ist, wie Abbildung 1 zeigt. Herausstechend sind Ferienwohnungen, Campingplätze und sonstige Betriebe im Beherbergungsbereich und Caterer/Betriebe der Gemeinschaftsverpflegung im Gastronomiebereich, während in Restaurants und Hotels die Belegschaft zu weitaus geringeren Teilen über 55 Jahre alt ist.

Abbildung 1: Altersverteilung der über 55-Jährigen im Gastgewerbe, Zablen aus Statistisches Bundesamt 2019, eigene Darstellung

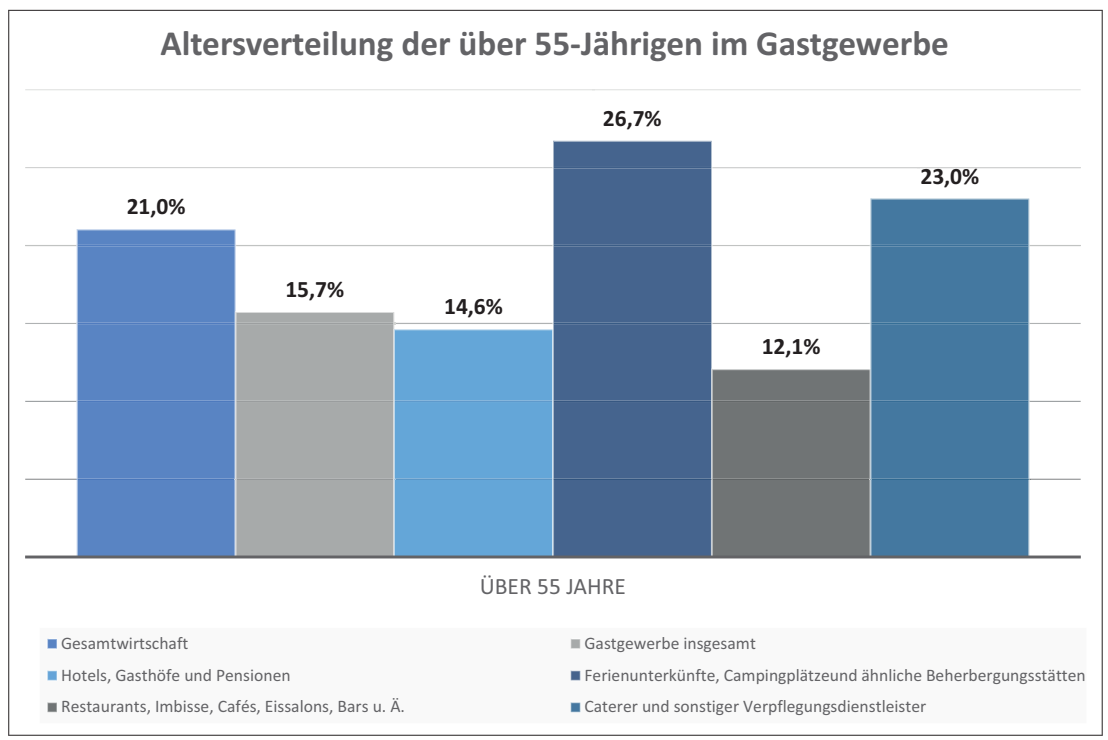

Der herausragende Unterschied zwischen diesen Betriebsformen und den übrigen betrifft die oben aufgeführten Arbeitsbelastungen: Die Arbeitszeiten sind im Schnitt wesentlich planbarer und liegen verstärkt im Tagesbereich, die Nachfrage ist vorhersehbarer, die Service- und Qualitätsansprü- 
che sind meist geringer. Für die Betrachtung der Potenziale arbeitslebenslanger Beschäftigung bietet sich daher vorerst an, nicht zwischen Beherbergung und Gastronomie, sondern Hotels/Restaurants und einfacher Beherbergung/Catering zu unterscheiden. Im Folgenden soll über die Ansprüche der Beschäftigten an die Arbeit im Lebensverlauf allgemein und im Gastgewerbe im Besonderen ergründet werden, welche Eigenschaften der zweiten Betriebsgruppe besonders ausschlaggebend für einen längeren Verbleib im Beruf sind.

\subsection{Zwischen Reputationsverlust und Vereinbarkeitsproblemen - Älterwerden im Gastgewerbe aus Sicht der Beschäftigten}

Dass die betriebliche Seite der Jugendzentriertheit der Branche dazu führt, dass besonders Hotels, Restaurants und Bars ,junge Teams“ als Qualitätsmerkmal vermarkten und es im Umkehrschluss zu einer Geringschätzung älterer Beschäftigter insbesondere im Kund"innenkontakt kommt, setzt sich in Einschätzungen auf der Seite der Beschäftigten fort. Bereits in jungen Jahren wollen viele den Beruf nicht ihr Leben lang ausüben, sondern setzen auf die brancheninternen Aufstiegschancen, die aufgrund der hierarchischen Struktur zahlreich und relativ schnell zu erlangen sind. In Interviews mit Beschäftigten begegnet einem die geteilte Einschätzung, dass man im Alter von 35 eine Führungsposition erlangt haben sollte oder den aktiven Dienst verlassen müsse (Guhlemann \& Krüger 2018). Tatsächlich zeigen Alterskurven für die Branche einen Abfall der Beschäftigtenzahl ab jenem Alter (Schlote-Sautter et al. 2018). In einer qualitativen Studie ließen sich als Ausstiegsgründe vor allem die Wahrnehmung einer Perspektivlosigkeit, insbesondere nach vielen brancheninternen Wechseln, verbunden mit der Wahrnehmung attraktiverer Möglichkeiten außerhalb der Branche und der Problematisierung betriebsübergreifend wiederkehrender Führungsmängel, Vereinbarkeitsprobleme in Familien- oder Qualifizierungsphasen und gesundheitliche Probleme identifizieren. Fälle von Altersdiskriminierung verbunden mit unfreiwilligem Verantwortungsentzug werden sogar auf Führungsebene und im Alter von 45-55 Jahren berichtet, ebenso erscheint eine Stundenreduzierung in Familienphasen unmöglich oder ist mit starken Reputationseinbußen versehen (Krüger \& Guhlemann 2018). Das grundlegende Dilemma ist, dass die prestigeträchtigeren, abwechslungsreicheren und interessanteren Arbeitstätigkeiten im Gastgewerbe gemeinhin in reputablen Häusern in den Abendstunden stattfinden und mit ausgedehnten, schlecht planbaren Arbeitszeiten einhergehen. Dies stellt Beschäftigte vor die Wahl zwischen hochwertiger Arbeit, die 
mit ihren Bedürfnissen kollidiert und einfachen Tätigkeiten, die familienund altersgerechter ist. Bereits eine feste Partnerschaft mit einer/einem Partner“in mit Tagesarbeitszeiten ist z.B. für Beschäftigte in einem Gourmet-Restaurant kaum aufrechtzuerhalten - ein Grund, warum viele Beschäftigte in der Phase der Paarbildung aus der Branche ausscheiden. Aber selbst für anpassungsfähigere Beschäftigte wird Altern im Gastgewerbe durch die inhärente Jugendkultur in den hochwertigeren Betrieben erschwert, die zu einer Geringschätzung „älterer“ Beschäftigter ohne Führungsposition führt, wobei „Alter“ in diesem Fall einigen Interviewaussagen zufolge bereits mit Mitte 30 beginnt. Die Geringschätzung von Erfahrungswissen und Fähigkeiten kann eine starke Quelle psychischer Belastung sein. Die Arbeit in weniger angesehenen Betrieben wie Kantinen wird unabhängig von der Position bei vielen Beschäftigten als gefühlter Abstieg, als dequalifizierend und eintönig wahrgenommen. Der Branchenausstieg ist dann für viele die bessere Alternative.

Hier scheinen Optimierungsmöglichkeiten in der betrieblichen Personalpolitik offensichtlich und potenzialträchtig, um den Berufsverbleib zu erhöhen. Doch auch unter Idealbedingungen kann eine Eignung der Branche für ein problemloses Älterwerden im Beruf bezweifelt werden. So zeigt eine Metastudie über den Wandel berufsbezogener Motive im Lebensverlauf starke Verschiebungen von intrinsischen Wachstumsmotiven in der Jugend (Abwechslung, Sozialklima, Lernförderlichkeit) über ökonomische Motive in der Lebensmitte (Einfluss, Geld, Karriere, Status) hin zu affektiven Motiven und Generativitätsmotiven im Alter (Bedeutsamkeit, Wertschätzung, interessante Arbeitsinhalte) (Grube 2009). Generell kann eine Zunahme von Bedürfnissen nach Stabilität und Planbarkeit im Erwerbsverlauf konstatiert werden, die mit Verschiebungen in der Wertigkeit von Lebensinteressen, wie Familiengründung oder Regeneration zusammenhängt, und die bereits nach „wenigen Jahren in flexibilisierten Arbeitszusammenhängen" auftreten können (Pongratz 2004, S. 41). Da sich der betriebliche Status im Gastgewerbe stark auf die Wahrnehmung der Arbeitsqualität auswirkt, stellen Übergänge in einfache Betriebe für viele Beschäftigte einen Abstieg dar (Guhlemann \& Krüger 2018), der besonders mit den beherrschenden Motiven der Lebensmitte nicht vereinbar ist. Mit Hinblick auf diese Befunde überrascht es wenig, dass Übergänge in Kantinen oder einfachere Beherbergungsbetriebe, obgleich sie Vereinbarkeitsprobleme lösen und gesundheitlich entlastend sein können, nur für einen Teil der Beschäftigten infrage kommen, nämlich für diejenigen, die von jeher stärker ausgeprägte Sicherheitsmotive als Statusorientierung angaben. 
Abbildung 2: Arbeitsfähigkeit im Gastgewerbe im biografischen Wandel, Quelle: eigene Darstellung

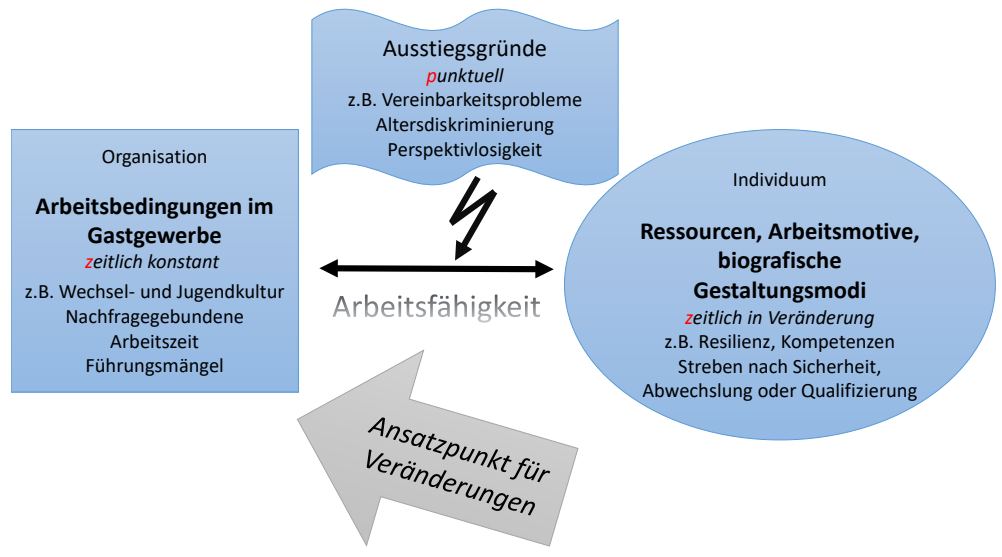

Wie in Abbildung 2 dargestellt, kann das Hauptproblem des Alterns im Gastgewerbe aus Beschäftigtensicht damit beschrieben werden, dass die (stabilen) Arbeitsbedingungen für die meisten Beschäftigten nur zu den Bedürfnissen früher Lebensphasen passen. Bemühungen um Beschäftigtenbindung sollten daher eine Adaptivität der Arbeitsbedingungen an die biografischen Veränderungen und unterschiedlichen Lebensphasen anstreben.

3. Probleme für einen längeren Berufsverbleib im Gastgewerbe und Ansätze zu deren Lösung

Aus den beschriebenen Verflechtungen von Arbeitsbedingungen und individuellen Lebenslagen können zentrale Problemkonstellationen identifiziert werden, die im Gastgewerbe einen langfristigen Berufsverbleib erschweren:

1. Kleinbetrieblichkeit erschwert ein strukturiertes Personalmanagement In den inhabergeführten Kleinbetrieben des Gastgewerbes fehlen häufig die Ressourcen und das ,Know-how' für eine strategische Personalentwicklung.

2. Konkurrenzdruck fördert Arbeitszeitüberschreitungen

Die hohe Konkurrenz zwischen den Kleinbetrieben bedingt die direkte Übertragung der Nachfragezeiten, die verbunden mit Perioden der 
Vor- und Nachbereitung häufig acht Stunden übersteigen, auf die Arbeitszeiten der Beschäftigten.

3. Personalmangel begünstigt Arbeitsbelastungen

Ein Fachkräfte- und Nachwuchsmangel verstärkt die Belastungen der verbleibenden Fachkräfte zum einen durch eine eventuelle Unterbesetzung und zum anderen durch die Notwendigkeit, Erfahrungsdefizite von Vertretungskräften auszugleichen.

4. Jugendliche Branchenkultur begünstigt Altersdiskriminierung Die Vermarktung eines, jungen Teams' als Konkurrenzkriterium führt aufseiten der Beschäftigten, Führungskräfte und Kund*innen zu einer Geringschätzung älterer Beschäftigter.

5. Gewandelte Anforderungen an Arbeit im Lebensverlauf sind nicht mit Karrieren vereinbar

Die aktuellen Arbeitsbedingungen im Gastgewerbe passen vordergründig zu den Motivlagen jüngerer Beschäftigter, bzw. lassen sich in frühen Lebensphasen besser tolerieren als in späteren.

6. Wechselkultur erleichtert Branchenausstiege

Die Normalität von Betriebswechseln in der Branche führt auf betrieblicher Seite zu einer Vernachlässigung von Bemühungen um Mitarbeiterbindung und baut auf der Seite der Beschäftigten Hemmschwellen gegen betriebsinterne wie -externe Wechsel ab.

7. Lebensphasengerechte Personalentwicklung ist noch stark ausbaufähig In den letzten Jahren sind trotz anhaltendem bis steigendem Mangel an Fachkräften und Nachwuchs wenig Erfolge darin erzielt worden, die Arbeitsbedingungen in der Branche auch für ältere Beschäftigte attraktiv zu machen.

Gestaltungsansätze, die den genannten Herausforderungen begegnen sollen, müssen somit die personalpolitischen Gegebenheiten kleiner Betriebe berücksichtigen, auf Dienstleistungsarbeit anwendbar sein und diskontinuierliche Erwerbsverläufe, Wechselkultur sowie begrenzte Tätigkeitsdauern berücksichtigen. Hier wären auch intermediäre Akteure wie Verbände, Berufsgenossenschaft und Kammern in stärkerem Maße gefordert, die Einhaltung von Schutzstandards sicherzustellen und sich um eine Sensibilisierung von Führungskräften für die genannten Problemlagen zu bemühen. Konzepte zur Gestaltung diskontinuierlicher Erwerbsverläufe können auf individueller, betrieblicher oder überbetrieblicher Ebene ansetzen. Betriebliche Unterstützungsangebote sollten dabei lebensphasenspezifisch erfolgen: Während jüngere Beschäftigte in der Orientierungsphase durchaus vielfältige und flexible Angebote attraktiv finden, bedürfen Beschäftigte mit veränderten lebensweltlichen Anforderungen ein höheres Maß an Sta- 
bilität und Planbarkeit. Auch das mit dem Alter oft abnehmende Level an Wertschätzung der eigenen Arbeitsleistung konfligiert mit dem reziprok wachsenden Bedürfnis danach. Mit einer stärkeren Berücksichtigung der lebensphasengeprägten individuellen Bedürfnisse der Beschäftigten sollte eine entsprechende Personalpolitik Beschäftigungsfähigkeit, Demografieorientierung und Work-Life-Balance integrieren. Als wesentliche Handlungsfelder erscheinen hier Kompetenzerhalt und -förderung, Identifikation und Motivation sowie Wohlbefinden und Gesundheit (Rump et al. 2014). Betriebspolitische Einflusssphären liegen neben der Wahrnehmung individueller Interessen auch darin, Autonomie zu fördern und Überbelastungen zu mindern. Eine wesentliche Stellschraube ist dabei die Sensibilisierung und Qualifizierung der Inhaber*innen und Führungskräfte für lebensphasen- und gesundheitsgerechte Führung.

Um alternsgerechte Erwerbsverläufe zu ermöglichen, müssen bereits bei Jugendlichen Fähigkeiten und Kompetenzen der erwerbsbiografischen Gestaltung gefördert werden. „Berufsbiografische Gestaltungskompetenz“ (Kaufhold 2009) im Sinne einer Befähigung zur Realisierung der subjektiv optimalen Karriereentwicklung in Relation zu den lebenslauf- und arbeitsmarktbezogenen Möglichkeiten und Erfordernissen geht über bloße Reaktionen auf Flexibilitätserfordernisse und Anpassungen an berufliche und lebenslaufbezogene Situationen hinaus. Zur Entwicklung der notwendigen Kontrollüberzeugung sind sowohl Erfassung als auch Einordnung beruflicher und personeller Voraussetzungen sowie der Ziele und Möglichkeiten geboten. Die Grundlagen für Reflexions- und Bilanzierungsfähigkeiten zur Herstellung von Sinnhaftigkeit erwerbsbiografischer Entwicklungen sollten daher die Praxis beruflicher Aus- und Weiterbildung ergänzen, die sich bislang hauptsächlich auf die Vermittlung von Fachkompetenzen konzentriert. Neben Handlungswissen zur Bewältigung erwerbsbiografischer Übergänge ist damit im Sinne eines Empowerments die Vorbereitung auf das gesamte Berufsleben unter Berücksichtigung erwartbarer Diskontinuitätsanforderungen angesprochen.

Es tritt deutlich hervor, dass alternsgerechte Arbeitsgestaltung nicht nur früh beginnen, sondern biografische Entwicklungen in ein Verhältnis zu den Arbeitsanforderungen setzen muss, um passgenaue Konzepte hervorzubringen. Hier wäre es zu begrüßen, wenn Ansätze und Erkenntnisse der Biografieforschung und der Gerontologie in einem noch stärkeren Maße in den arbeitsbezogenen Wissenschaften Berücksichtigung fänden, wozu mit den Konzepten lebensphasenbezogener Arbeitsgestaltung bereits ein Grundstein gelegt ist. 


\section{Literatur}

BA - Bundesagentur für Arbeit. (2019a). Der Arbeitsmarkt in Deutschland 2018. Abgerufen 26.05.2020, unter https://statistik.arbeitsagentur.de/Statistikdaten/De tail/201812/ama/heft-arbeitsmarkt/arbeitsmarkt-d-0-201812-pdf.pdf.

BA - Bundesagentur für Arbeit. (2019b). Die Arbeitsmarktsituation von Frauen und Männern 2018. Abgerufen 27.05.2020, unter https://statistik.arbeitsagentur. $\mathrm{de} /$ Statischer-Content/Arbeitsmarktberichte/Personengruppen/generische-Publi kationen/Frauen-Maenner-Arbeitsmarkt.pdf.

BIBB - Bundesinstitut für Berufsbildung. (2020). Datenreport zum Berufsbildungsbericht 2020. Abgerufen 29.05.2020, unter https://www.bibb.de/datenreport/de/ datenreport_2020.php.

Börsch-Supan, A. (2013). Mikro- und makroökonomische Dimensionen des demografischen Wandels. In: M. Hüther \& G. Naegele (Hrsg.), Demografiepolitik. Herausforderungen und Handlungsfelder (S.96-122). Wiesbaden: Springer Fachmedien.

Brandl, S., Guggemos, P. \& Matuschek, I. (2020). Altern in Betrieben als Herausforderung von Personalmanagement, Betriebsräten, Tarifparteien und Fachberatungsstellen. In: T. Freiling, R. Conrads, A. Müller-Osten \& J. Porath (Hrsg.), Zukünftige Arbeitswelten (S. 117-139). Wiesbaden: Springer.

DEHOGA - Der Hotel- und Gaststättenverband. (2018). Was ist dran an der Story von der hohen «Abbrecherquote»? Abgerufen 29.05.2020, unter https://www.de hogabw.de/informieren/dehoga-nachrichten/2018/zahlen-und-fakten-zur-sogena nnten-abbrecherquote.html.

DEHOGA - Der Hotel- und Gaststättenverband. (2020). Ausbildungsbilanz des Gastgewerbes 1991 bis 2019. Abgerufen 27.05.2020, unter https://www.dehoga-b undesverband.de/zahlen-fakten/ausbildungszahlen/.

Dettmann, E., Fackler, D., Müller, S., Neuschäffer, G., Slavtchev, V., Leber, U. \& Schwengler, B. (2019). Fehlende Fachkräfte in Deutschland - Unterschiede in den Betrieben und mögliche Erklärungsfaktoren: Ergebnisse aus dem IAB-Betriebspanel 2018. IAB-Forschungsbericht, 10.

Deutsche Rentenversicherung. (2019). Rentenatlas 2019. Die Deutsche Rentenversicherung in Zahlen, Fakten und Trends. Abgerufen 03.06.2020, unter https://fs. flockusercontent.com/347382/09c63651591177624e49c3cd? Expires=1591178237 \&Signature=GR8dvA05S0RnF2d7MaMR67RJYf7tOHY7Fnb1dHLL0IVtC3aHu IrfAVQYJq7DtvQKxqi9lvdfs6wE1xGEco8OVlJZZE1ZlYjxGNTa9a4tkTiQfhKJX -Rg6QBTxg8zcB7Wwdqtq2rW--kqBeF1lTBIZukCqPbcuzQyEeIHyoYyXmftqe0 HtfypdpbYK7KB2XkkAzFRgVFAfugCs5qL15g ZynQdhudqt3H-cnSw2aP3AwX 9MjarLF 8-VtxM5Ocs72d5TVMtknx2IC6Wv3W VFcObFb3Zh8wN Qf52npSd CXng5YQkqzYTjq1D70V9SkyRN3e6nA9cigScjIJeEPgrgg_\&Key-Pair-Id=APK AJMN6OEFOLBEBMIJA.

DGB Bundesvorstand. (2019). Ausbildungsreport 2019. Ausbildung 4.0 // digital. Lernen. Gemeinsam. Entwickeln. Abgerufen 27.05.2020, unter https://www.dgb .de/++co++9cafb4fe-c996-11e9-b8a9-52540088cada. 
DIHK- Deutscher Industrie- und Handelskammertag e.V. (2019). Ausbildung 2019. Ergebnisse einer DIHK-Online-Unternehmensbefragung. Abgerufen 27.05.2020, unter https://www.dihk.de/resource/blob/10074/a34c93fa0d1ea9989fe37a357e9b d3dc/dihk-umfrage-ausbildung-2019--data.pdf.

Frerichs, F. (2019). Altern in der Erwerbsarbeit: Laufbahngestaltung bei begrenzter Tätigkeitsdauer. In S. Kauffeld \& D. Spurk (Hrsg.), Handbuch Karriere und Laufbahnmanagement (S. 893-912). Berlin: Springer-Verlag.

Grube, A. (2009). Alterseffekte auf die Bedeutung berufsbezogener Motive und die Zielorientierung. Diss. Münster: Westfälische Wilhelms-Universität zu Münster (Westf.).

Guhlemann, K. (2020). Prävention 4.0 im Prozess digitaler Arbeitsgestaltung. In: A. Georg, K. Guhlemann \& G. Peter (Hrsg.), Humanisierung der Arbeit 4.0. Prävention und Demokratie in der digitalisierten Arbeitsgesellschaft (S. 113-138). Hamburg: VSA Verlag.

Guhlemann, K. \& Krüger, F. (2018). Immer auf dem Sprung. Typische Erwerbsbiografien und kritische Berufsphasen im Gastgewerbe. In: E. Beerheide, A. Georg, A. Goedicke, C. Nordbrock, K. Seiler (Hrsg.), Gesundheitsgerechte Dienstleistungsarbeit (S. 107-132). Wiesbaden: Springer VS.

Guhlemann, K., Krüger, F., Georg, A. \& Katenkamp, O. (2016). Gestaltungsoptionen von Berufskarrieren im Gastgewerbe - Kontinuierlich wechselhaft. In: F. Frerichs (Hrsg.), Altern in der Erwerbsarbeit. Perspektiven der Laufbahngestaltung (S. 313-326). Wiesbaden: Springer VS.

Hasselhorn, H. M. \& Ebener, M. (2018). Die differenzierte Rolle von Gesundheit für die Erwerbsteilhabe im höheren Erwerbsalter - eine Diskussion anhand des „Iida-Denkmodells zu Arbeit, Alter und Erwerbsteilhabe”. In: E. M. Hohnerlein, S. Hennion \& O. Kaufmann (Hrsg.), Erwerbsverlauf und sozialer Schutz in Europa (S. 215-223). Berlin: Springer.

Ilmarinen, J. (2012). Förderung des aktiven Alterns am Arbeitsplatz. Abgerufen 03.06.2020, unter https://www.bgm-ag.ch/files/public/literatur/pdf/foerderung-d es-aktiven-alterns-am-arbeitsplatz.pdf.

Kaufhold, M. (2009). Berufsbiografische Gestaltungskompetenz. In: A. Bolder \& R. Doboschat (Hrsg.), Eigen-Sinn und Widerstand. Kritische Beiträge zum Kompetenzentwicklungsdiskurs (S. 220-228). Berlin: Springer.

Kistler, E. (2008). „Alternsgerechte Erwerbsarbeit“. Ein Überblick über den Stand von Wissenschaft und Praxis. Düsseldorf: Hans-Böckler-Stiftung.

Kistler, E., Holler, M. \& Schneider, D. (2019). Alter(n)sgerechte Arbeitsbedingungen und Lebenslagen - Fiktionen und Fakten. In: W. Schneider \& S. Stadelbacher (Hrsg.), Der Altersübergang als Neuarrangement von Arbeit und Leben. Kooperative Dienstleistungen für das Alter(n) in Vielfalt (S. 89-113). Wiesbaden: Springer VS.

Krüger, F. \& Guhlemann, K. (2018). Arbeit und Arbeitsbedingungen im Gastgewerbe. In: E. Beerheide, A. Georg, A. Goedicke, C. Nordbrock, K. Seiler (Hrsg.). Gesundheitsgerechte Dienstleistungsarbeit (S. 79-104). Wiesbaden: Springer VS.

Naegele, G. (2005). Nachhaltige Arbeits- und Erwerbsfähigkeit für ältere Arbeitnehmer. WSI-Mitteilungen 4/2005, 214-219. 
Naegele, G. (2000). Trends und Herausforderungen - Einführung in den Kongress „Altern und Arbeit“. In: C. von Rothkirch (Hrsg), Altern und Arbeit: Herausforderung für Wirtschaft und Gesellschaft Beitrage, Diskussionen und Ergebnisse eines Kongresses mit internationaler Beteiligung. Berlin: Ed. Sigma.

Pongratz, H. (2004). Die Verunsicherung biographischer Perspektiven. Erwerbsbiographien zwischen Normalitätserwartungen und Flexiblisierungsdruck. In: F. Behringer, A. Bolder, R. Klein, G. Reutter \& A. Seiverth (Hrsg.), Diskontinuierliche Erwerbsbiographien. Zur gesellschaftlichen Konstruktion und Bearbeitung eines normalen Phänomens (S. 27-45). Baltmannsweiler: Schneider Verlag Hohengehren.

Rump, J., Wilms, G. \& Eilers, S. (2014). Die Lebensphasenorientierte Personalpolitik. Grundlagen und Gestaltungstipps aus der Praxis für die Praxis. In: J. Rump \& S. Eilers (Hrsg.), Lebensphasenorientierte Personalpolitik. Strategien, Konzepte und Praxisbeispiele zur Fachkräftesicherung (S.3-69). Berlin: Springer Gabler.

Schlote-Sautter, B., Herter-Eschweiler, R., Keller, S. (2018). Beschäftigungs- und Betriebsstrukturen im Gastgewerbe. In: E. Beerheide, A. Georg, A. Goedicke, C. Nordbrock, K. Seiler (Hrsg.), Gesundheitsgerechte Dienstleistungsarbeit (S. 43-78). Wiesbaden: Springer VS.

Schneider, W. \& Stadelbacher, S. (2019). Der Altersübergang als gesellschaftliches Problem und als Gestaltungsaufgabe - Einführende Überlegungen zu einer risikoreichen Lebensphase. In W. Schneider \& S. Stadelbacher (Hrsg.), Der Altersübergang als Neuarrangement von Arbeit und Leben. Kooperative Dienstleistungen für das Alter(n) in Vielfalt (S. 1-15). Wiesbaden: Springer VS.

Schröder-Kunz, S. (2019). Generationen (gut) führen. Altersgerechte Arbeitsgestaltung für alle Mitarbeitergenerationen. Wiesbaden: Springer Gabler.

Stadtmüller, S. (2016). Demografischer Wandel und unpopuläre Reformen. Der Einfluss von Informationen auf die Bewertung der Rente mit 67. Wiesbaden: Springer VS.

Statistik der Bundesagentur für Arbeit. (2019). Situation von Älteren. Abgerufen 29.05.2020, unter https://statistik.arbeitsagentur.de/Statischer-Content/Arbeitsm arktberichte/Personengruppen/generische-Publikationen/Aeltere-amArbeitsmar kt.pdf.

Statistisches Bundesamt. (2019). Statistisches Jahrbuch. Deutschland und Internationales. Abgerufen 29.05.2020, unter https:/www.destatis.de/DE/Themen/Quer schnitt/Jahrbuch/statistisches-jahrbuch-2019-dl.pdf?_blob=publicationFile.

Varekamp, I., van Dijk, F., Kroll, L. (2013). Workers with a chronic disease and work disability. Problems and Solutions. Bundesgesundheitsblatt Gesundheitsforschung Gesundheitsschutz, 56, 406-414.

\section{Referenzen}

1 Zwischen 2008 und 2018 stieg die Erwerbsbeteiligung von über 55-Jährigen von 54 auf $72 \%$ (Statistisches Bundesamt 2019, S. 370). 
2 Zumal hier aufgrund der Nichtexistenz „des einen Alters“ bereits Definitionsprobleme einsetzen, wann dies sein soll.

3 Nach gängiger arbeitswissenschaftlicher Erkenntnis beeinflussen sich beide Bereiche des Lebens wechselseitig und bieten sowohl Ressourcen als auch Belastungen, die in den anderen Bereich hineinwirken (vgl. u.a. Schröder-Kunz 2019,S. 64).

4 Der Fluktuationskoeffizient ist eine Maßzahl für den Personalumschlag, konkret das Verhältnis aus Zu- und Abgängen zum Personalbestand. Mit 0,696 erreicht das Gastgewerbe den doppelten Wert der Gesamtwirtschaft $(0,33)$ und übersteigt den der Dienstleistungsbranche $(0,373)$ erheblich. 
